\title{
ON NILPOTENT GROUPS OF AUTOMORPHISMS OF KLEIN SURFACES
}

\author{
EMILIO BUJALANCE AND GRZEGORZ GROMADZKI \\ (Communicated by Irwin Kra) \\ In memory of Andrzej Sobkowiak
}

\begin{abstract}
The nilpotent group of automorphisms of a bordered Klein surface $X$ of algebraic genus $q \geq 2$ is known to have at most $8(q-1)$ elements. Moreover this bound is attained if and only if $\mathbf{q}-1$ is a power of 2 . In this paper we prove that if $X$ is nonorientable and $q \geq 3$ then the bound in question can be sharpened to $\mathbf{4 q}$ which gives a negative answer to a conjecture of May [16]. We also solve another problem of May [16] finding bounds for the $p$-groups of automorphisms of Klein surfaces.
\end{abstract}

\section{INTRODUCTION}

Let $X$ be a compact Klein surface of algebraic genus $\mathbf{q} \geq 2$ and let $G$ be a group of its automorphisms. Then $|G| \leq 84(q-1)$. If a surface $X$ has nonempty boundary then this bound can be strengthened to $12(\mathbf{q}-1)$. Although it is attained for infinitely many values of $\mathbf{q}[6,7,8,12,14,15,16,17,19]$, it was shown recently by May [16] that no nilpotent group of automorphisms of a bordered Klein surface of algebraic genus $q$ has order 12(q-1). It was also shown in [16] that $8(\mathbf{q}-1)$ is the bound in this case as well as that a nilpotent group for which it is attained must be a 2-group. Moreover this bound is attained for every $q-1$ being a power of 2 . Nevertheless the only one surface constructed by May is nonorientable (a real projective plane with two holes having the dihedral group of order 8 as a group of automorphisms).

Having a Klein surface with a nilpotent group of automorphisms of maximal possible order one can produce an infinite collection of orientable surfaces having this property, while no general method of producing nonorientable ones is known. This led May in [16] to ask if the bound $8(\mathbf{q}-1)$ for the order of a 2-group of automorphisms of a compact nonorientable bordered Klein surface

Received by the editors June 1, 1988 and, in revised form, April 25, 1989. $20 \mathrm{~F} 18$.

1980 Mathematics Subject Classification (1985 Revision). Primary 30F10; Secondary 20F05,

Key words and phrases. Klein surfaces, algebraic genus, automorphism, nilpotent group.

The first author was partially supported by CAICYT.

The second author was supported by the postdoctoral grant of the Spanish Ministry of Education and Sciences. 
of algebraic genus $\mathbf{q} \geq 3$ can be improved in a former version and conjecture in the revised one that the answer to this question is "no." We show in this paper that this conjecture is false proving that a nilpotent group of automorphisms of a nonorientable bordered Klein surface of genus $\mathbf{q} \geq 3$ has at most $4 \mathbf{q}$ elements. Moreover we show that this bound is attained for every $\mathbf{q}$ being a power of 2, and dihedral groups turn out to be the corresponding groups of automorphisms. From our results on presentations of 2-groups it follows also that a nilpotent group of automorphisms of a nonorientable Riemann surface of algebraic genus $\mathbf{q} \geq 2$ has at most $8(\mathbf{q}-1)$ elements. Thus the Zomorrodian result on nilpotent groups of orientation-preserving automorphisms of Riemann surfaces [22] cannot be generalized to the nonorientable case. This fact is in marked contrast with the corresponding results for the maximal groups of automorphisms of compact Klein surfaces for which the bounds $84(\mathbf{q}-1)$ (in a case of surfaces without boundary [20]) and 12(q-1) (in a case with boundary) are attained both in orientable and in nonorientable cases.

Finally we solve another problem posed by May in [16] of determining the best upper bound for the order of a $p$-group of automorphisms of a Klein surface of genus $\mathbf{q} \geq 2$.

\section{Preliminaries}

We will prove the announced results by means of NEC-groups. An NECgroup is a discrete subgroup $\Gamma$ of isometries $\mathfrak{G}$ of the non-Euclidean plane $\mathbf{C}^{+}$ (including those which reverse orientation-reflections and glide reflections) with compact quotient space $\mathbf{C}^{+} / \Gamma$. Let $\mathfrak{G}^{+}$denote the subgroup of index 2 in $\mathfrak{G}$ consisting of orientation preserving isometries. An NEC-group $\Gamma$ contained in $\mathfrak{G}^{+}$is called a Fuchsian group, and a proper NEC-group in the other case. In what follows $\Gamma^{+}=\Gamma \cap \mathfrak{G}^{+}$is the canonical Fuchsian subgroup of an NEC-group $\Gamma$.

Macbeath and Wilkie [10,21] associated to every NEC-group a signature that has the form

$$
\left(g ; \pm ;\left[m_{1}, \ldots, m_{r}\right],\left\{\left(n_{11}, \ldots, n_{1 s_{1}}\right), \ldots,\left(n_{k 1}, \ldots, n_{k s_{k}}\right)\right\}\right)
$$

and determines the algebraic structure of the group.

The numbers $m_{i}$ are called proper periods, the brackets $\left(n_{i 1}, \ldots, n_{i s_{i}}\right)$ the period cycles and $g \geq 0$ is called orbit genus. The group with signature (2.1) has the presentation with the following generators

$$
\begin{aligned}
& \text { (i) } x_{i}, \quad i=1, \ldots, r, \\
& \text { (ii) } c_{i j}, \quad i=1, \ldots, k, \quad j=0, \ldots, s_{i}, \\
& \text { (iii) } e_{i}, \quad i=1, \ldots, k, \\
& \text { (iv) } a_{i}, b_{i}, \quad i=1, \ldots, g \quad \text { (if the sign is }+ \text { ) } \\
& d_{i}, \quad i=1, \ldots, g \quad \text { (if the sign is }- \text { ) }
\end{aligned}
$$

subject to the relations

$$
\text { (i) } x_{i}^{m} i=1, \quad i=1, \ldots, r \text {, }
$$


(ii) $c_{i s_{i}}=e_{i}^{-1} c_{i 0} e_{i}, \quad i=1, \ldots, k$,

(iii) $c_{i, j-1}^{2}=c_{i, j}^{2}=\left(c_{i, j-1} c_{i j}\right)^{n} i j=1, \quad i=1, \ldots, k, j=1, \ldots, s_{i}$,

(iv) $x_{1} \ldots x_{r} e_{1} \ldots e_{k} a_{1} b_{1} a_{1}^{-1} b_{1}^{-1} \ldots a_{g} b_{g} a_{g}^{-1} b_{g}^{-1}=1$ (if the sign is + ) $x_{1} \ldots x_{r} e_{1} \ldots e_{k} d_{1}^{2} \ldots d_{g}^{2}=1$ (if the sign is - ).

In what follows these generators are said to be the canonical generators of $\Gamma$. Every NEC-group has a fundamental region associated, whose area depends only on the group and not on the chosen region. It is given by

$$
\mu(\Gamma)=2 \pi\left(\alpha g+k-2+\sum_{i=1}^{r}\left(1-1 / m_{i}\right)+\sum_{i=1}^{k}\left(1-1 / n_{i j}\right) / 2\right),
$$

where $\alpha=1$ if the sign is - and $\alpha=2$ in the other case.

It is known that a group $\Gamma$ with presentation (2.2) can be realized as an NEC-group with signature (2.1) if and only if the right-hand side of (2.3) is greater than 0 .

If $\Gamma$ is a subgroup of finite index in an NEC-group $\Lambda$, then it is an NECgroup itself and the following Riemann-Hurwitz formula holds

$$
[\Lambda: \Gamma]=\mu(\Gamma) / \mu(\Lambda) \text {. }
$$

An NEC-group with signature

$$
(g ; \pm ;[-],\{(-), . . k,(-)\})
$$

will be called a surface group of genus $g$ with $k$ boundary components orientable or nonorientable accordingly, as the sign is + or - . If $k>0$ or the sign is - then the number $\mathbf{q}=\alpha g+k-1$ is called the algebraic genus of $\Gamma$. It equals the algebraic genus of the corresponding Klein surface $X=\mathrm{C}^{+} / \Gamma$. If $k=0$ and the sign is + then the algebraic genus $\mathbf{q}$ equals the topological genus $g$.

It is known [18] that a compact (bordered) Klein surface of genus $\mathbf{q} \geq 2$ can be represented as $\mathbf{C}^{+} / \Gamma$, where $\Gamma$ is a (bordered) surface group of algebraic genus $\mathbf{q}$. Moreover given a surface so represented, a finite group $G$ is a group of its automorphisms if and only if there exists an NEC-group $\Lambda$ and a homomorphism $\theta$ from $\Lambda$ onto $G$ having $\Gamma$ as the kernel [11]. Such a homomorphism is said to be a (bordered or unbordered, orientable or nonorientable according as $k>0$ or $k=0$ and $\Gamma$ has the sign + or - respectively) surface-kernel homomorphism, a group $G$ so represented a surface-kernel factor group of $\Lambda$, while $\Lambda$ is said to admit $G$ as a surface-kernel factor. In what follows by a surface (surface group) we will mean unbordered surface (surface group).

In order to state the facts which will be necessary for our considerations we need some more notations. Given an NEC-group $\Gamma$ and a subgroup $\Gamma_{1}$, a canonical generator of $\Gamma$ is said to be proper (with respect to $\Gamma_{1}$ ) if it does not belong to $\Gamma_{1}$. An element of $\Gamma$ expressible as a composition of proper generators of $\Gamma$ is called a word of $\Gamma$ (with respect to $\Gamma_{1}$ ). Finally a given 
word is orientable if it preserves orientation of $\mathrm{C}^{+}$and nonorientable in the other case. With the above notations we have the following results.

Theorem 2.1 [4, 9]. Let $\Gamma_{1}$ be a subgroup of an NEC-group $\Gamma$ of finite index $N$. Then

(1) If $N$ is odd then $\Gamma_{1}$ is orientable if and only if $\Gamma$ is.

(2) If $N$ is even then $\Gamma_{1}$ is nonorientable if and only if a glide reflection of the canonical generators of $\Gamma$ or a nonorientable word in $\Gamma$ belongs to $\Gamma_{1}$.

Theorem 2.2 [3]. An NEC-group $\Gamma$ admits a bordered surface subgroup as a normal subgroup of finite index if and only if it has a signature with an empty period cycle or with a period cycle with two consecutive periods equal to 2.

\section{ON GENERATORS AND RELATIONS OF FINITE NILPOTENT GROUPS}

Lemma 3.1. Let $\left\langle x, y, z \mid x^{2}, y^{2}, z^{2},(x y)^{2},(y z)^{2},(x z)^{k}\right\rangle$ be the presentation of the dihedral group $G$ of order $2 k$. Then $k$ is even and $y=(x z)^{k / 2}$.

Proof. $x y x^{-1}=y$ and $z y z^{-1}=y$. Thus a subgroup $H$ generated by $y$ has order 2 and is a normal subgroup of $G$. Thus $\widetilde{G}=G / H$ has order $k$. On the other hand $\widetilde{G}$ is a factor group of the group with presentation $\left\langle x, z \mid x^{2}, z^{2},(x z)^{k}\right\rangle$. As a result the relation $(x z)^{k / 2}$ holds in $\widetilde{G}$, otherwise it would have the order $2 k$. Thus $(x z)^{k / 2}$ belongs to $H$, and so $y=(x z)^{k / 2}$ holds in the former group, as desired.

The importance of the next lemma lies in the fact that it shows that the approach, suggested in [16, Problem 2, p. 292], for finding nonorientable Klein surfaces of algebraic genus $\mathbf{q} \geq 3$ with nilpotent group of automorphisms of order $8(\mathbf{q}-1)$ fails.

Lemma 3.2. A finite nilpotent group $G$ of order greater than 8 cannot be generated by three elements $A, B$, and $C$, of orders 2 such that $A B$ and $B C$ generate the whole group $G$ and $A B, B C$ and $A C$ have orders $2, k$ and $l$, respectively, where $k$ and $l$ are greater than 2 .

Proof. Assume, by the way of contradiction, that a group $G$ in question exists. A finite nilpotent group is the product of its Sylow subgroups and $G$ is generated by elements of order 2. Thus $G$ is a 2-group, say of order $2^{N}$, and $k=2^{m}$, $l=2^{n}$, where $m \geq 2$ or $n \geq 2$. Changing the role of the generators $A$ and $B$, if necessary, we can assume that $k \leq l$. Let $N=4$. Then it is easy to see that among 14 groups of order 16 there is no such group. So $N \geq 5$. In all the cases considered below we will assume that $N$ is minimal.

First assume then that $k=l=4$, and let $H$ be a normal subgroup of $G$ of order 2. Let $\widetilde{G}=G / H$. By the minimality of $G A, B, C, A B,(B C)^{2}$, or $(A C)^{2}$ belong to $H$. But then this element would become in $\widetilde{G}$ a relation that collapses it to a group of order $\leq 8$, a contradiction.

Now let $k=4$ and $l \geq 8$. Consider, as in the first case, a normal subgroup $H$ of $G$ of order 2 and let $\widetilde{G}$ be the quotient. By the minimality of $G$ at 
least one of $A, B, C, A B$, or $(B C)^{2}$ belongs to $H$. But if $A, C$ or $A B$ belonged then it would produce in $\widetilde{G}$ a relation collapsing it to a group of order $\leq 8$. If $B$ belonged to $H$ then since $H$ is normal in $G,(B C)^{2}$ would be a relation in the former group. Finally if none of the elements considered before belonged to $H$ then by the minimality of $G,(B C)^{2}$ would belong to $H$. But then it would collapse $\widetilde{G}$ to a dihedral group with the presentation

$$
\left\langle\widetilde{A}, \widetilde{B}, \widetilde{C} \mid \widetilde{A}^{2}, \widetilde{B}^{2}, \widetilde{C}^{2},(\widetilde{A} \widetilde{B})^{2}, m(\widetilde{B} \widetilde{C})^{2},(\widetilde{A} \widetilde{C})^{s}\right\rangle,
$$

where $s=1$ or $s=\frac{1}{2}(\tilde{A}, \widetilde{B}$, and $\widetilde{C}$ are the images of $A, B$, and $C$, respectively, under the canonical projection). By Lemma 3.1 $\widetilde{B}=(\widetilde{A} \widetilde{C})^{s / 2}$, and so $\widetilde{B}(\widetilde{A} \widetilde{C})^{s / 2}=1$. Thus $B(A C)^{s / 2}$ belongs to $H$. As a result $B(A C)^{s / 2}=1$ or $B(A C)^{s / 2}=(B C)^{2}$. In the first case $C B=C(A C)^{s / 2}$ and so $B C$ has order 2, a contradiction, while in the second $B C=C(C A)^{s / 2}$ and thus $B C$ also has order 2, a contradiction once more.

Finally assume that $8 \leq k \leq l$. Let as before $H$ be a normal subgroup of $G$ of order 2 and let $\widetilde{G}$ be the corresponding quotient. If $C$ belonged to $H$ then $\widetilde{G}$ would be a group of order $\leq 4$. If $A$ were in $H$ then since $H$ is a normal subgroup of $G,(A C)^{2}$ would be a relation in $G$. Similarly for $B$. If $A B$ belonged to $H$ then the quotient would be a cyclic group generated by $\widetilde{B} \widetilde{C}$-the product of two elements of order 2 , a contradiction once more. Now since $k, l \geq 8, \widetilde{G}$ would be a group satisfying the hypothesis of the lemma.

This is a contradiction with the minimality of $G$.

Corollary 3.3. Let $G$ be a nilpotent bordered nonorientable surface-kernel factor of an NEC-group $\Lambda$ with signature $(0 ;+;[-],\{(2,2,2, k)\})$. Then $k$ is a power of 2 and $G$ is the dihedral group of order $2 k$.

Proof. Let $\Lambda$ and $G$ be groups in question and let $\theta$ be the corresponding homomorphism. The group $G$ is generated by elements of order 2 and so is a 2-group. Thus $k$ is a power of 2 , since otherwise a nontrivial power of $c_{3} c_{4}$ would be in $\operatorname{Ker} \theta$. It is easy to see that $\Lambda$ is generated by four elements $c_{0}$, $c_{1}, c_{2}$, and $c_{3}$ subject to the relations

$$
\left(c_{0} c_{1}\right)^{2}=\left(c_{1} c_{2}\right)^{2}=\left(c_{2} c_{3}\right)^{2}=\left(c_{0} c_{3}\right)^{k}=1 \text {. }
$$

First notice that $\theta\left(c_{0}\right) \neq 1$ and $\theta\left(c_{3}\right) \neq 1$, since otherwise $\Gamma$ would have nonempty period cycle by [5]. Moreover since $\Gamma$ has a boundary it contains one of the remaining reflections. Without loss of generality we can assume that $\theta\left(c_{1}\right)=1$. Then $\theta\left(c_{2}\right) \neq 1$, since otherwise $\Gamma$ has nontrivial period cycle by [5]. As a result

$$
\theta\left(c_{0}\right)=x, \quad \theta\left(c_{1}\right)=1, \quad \theta\left(c_{2}\right)=y, \quad \theta\left(c_{3}\right)=z,
$$

where $x, y$, and $z$ are elements of order 2 such that $y z$ and $x z$ have orders 2 and $k$, respectively.

Now we will show that if $\Gamma$ is nonorientable then the subgroup $H$ of $G$ generated by $x z$ and $y z$ equals the whole group $G$. First notice that $c_{0}, c_{2}$, 
$c_{3}$ are the only proper generators of $\Lambda$ with respect to $\Gamma$. Thus an element $w$ is a nonorientable word in $\Gamma$ if and only if it can be presented as a product of an odd number of these generators. But it is easy to see that such a word can be written as a product $u v$, where $u$ is a product of $\left(c_{0}, c_{3}\right),\left(c_{2}, c_{3}\right)$, their inverses and $v$ is one of the reflections, say $c_{0}$. Thus $\theta\left(c_{0}\right)$ belongs to $H$. But then since $\theta\left(c_{3}\right)=\theta\left(c_{0}\right) \theta\left(c_{0} c_{3}\right)$ and $\theta\left(c_{2}\right)=\theta\left(c_{2} c_{3}\right) \theta\left(c_{3}\right), \theta\left(c_{2}\right)$ and $\theta\left(c_{3}\right)$ also belong to $H$. So $H=G$ as desired.

Let $l$ be the order of $x y$. Then by the previous lemma $l=1$ or $l=2$. But in the first case $x=y$ and so $x z=y z$, a contradiction, while in the second $G$ is dihedral group of order $2 k$, as desired.

Corollary 3.4. None of NEC-group $\Lambda$ with signature $(0 ;+;[-],\{(2, k, l)\})$ admits a finite nilpotent group as a nonorientable surface-kernel factor.

Proof. Assume to get a contradiction that $G$ is a nilpotent nonorientable surface-kernel factor of a group $\Lambda$. Then by a result of Singerman [20] $G$ is generated by 3 elements $A, B$, and $C$ of order 2 whose products $A B, B C$, and $A C$ have orders $2, k$, and $l$, respectively and in addition $A B$ and $B C$ generate the whole group $G$. This is a contradiction of Lemma 3.2.

\section{MAIN RESULTS ON 2-GROUPS \\ OF AUTOMORPHISMS OF COMPACT NONORIENTABLE KLEIN SURFACES}

Theorem 4.1. Let $G$ be a nilpotent group of automorphisms of a nonorientable bordered Klein surface of algebraic genus $q \geq 3$. Then $|G| \leq 4 \mathbf{q}$. Moreover this bound is attained for every $\mathbf{q}$ being a power of 2 and the corresponding group of automorphisms is the dihedral group.

Proof. Let $G$ be a finite nilpotent group of automorphisms of a compact nonorientable bordered Klein surface of algebraic genus $\mathbf{q} \geq 3$. Then $G=\Lambda / \Gamma$, where $\Lambda$ is a proper NEC-group and $\Gamma$ is a nonorientable bordered surface group of algebraic genus q, i.e. $\mu(\Gamma)=2 \pi(\mathbf{q}-1)$. Let $|G|=N$. First we will show that $\mu(\Lambda) \geq \pi((N-4) / 2 N)$.

Let $\Lambda$ have signature (2.1). By Theorem $2.2 k>0$. If $k>2$ then $\mu(\Lambda) \geq$ $2 \pi$. If $k=2$ and either $g>0$ or $\Lambda$ has a proper period then $\mu(\Lambda) \geq \pi$, while in the other case, one of the period cycles is nonempty and then clearly $\mu(\Lambda) \geq \pi / 2$. Assume thus that $k=1$.

If the period cycle is empty then from Theorem 2.1 it follows that the sign is - . If $g \geq 2$ then $\mu(\Lambda) \geq 2 \pi$. If $g=1$ then $r>0$, since otherwise $\mu(\Lambda)=0$. But in this case $\mu(\Lambda) \geq \pi$.

If the period cycle is nonempty then by Theorem 2.2 at least two of its periods are equal to 2 . If $g \geq 1$ then $\mu(\Lambda) \geq \pi / 2$.

Thus we can assume that $\Lambda$ has a signature $\left(0,+,\left[m_{1}, \ldots, m_{r}\right]\right.$, $\left.\left\{\left(n_{1}, \ldots, n_{s}\right)\right\}\right)$. Notice first that since $G$ is a product of its Sylow subgroups all $n_{i}$ are powers of 2 , otherwise $\Gamma$ would have a nontrivial period [1,2]. By Theorem $2.2 s \geq 2$ and if $s=2$ then $n_{1}=n_{2}=2$. If in addition $r \geq 2$ then 
$\mu(\Lambda) \geq \pi$. Assume then that $r=1$. If $m=2$ then $\mu(\Lambda)=0$. If $m=3$ then $x_{1}=e^{-1}$ and since $G$ is a direct product of its Sylow subgroups the images of $e$ and $c_{i}$ commute for all $i$. In particular the relation $e c_{0} e^{-1}=c_{2}$ in $\Lambda$ implies $c_{0}=c_{2}$ in the factor group. Now since $\Gamma$ is a bordered surface subgroup of $\Lambda$, a canonical reflection of $\Lambda$ belongs to $\Gamma$. If $c_{0}$ belongs to $\Gamma$ then $c_{1}$ is the only orientation reversing proper generator of $\Lambda$ and it is easy to see that it and other proper generators of $\Lambda$ cannot produce a nonorientable word. In fact using the relation $x_{1}=e^{-1}$ in $\Lambda$ and the fact that the image of $x_{i}$ and $c_{i}$ in $G$ commute one can choose such a word $w$ in the form $w=x_{1}^{k} c_{1}$. But then $c_{1}$ would equal $x_{1}^{k}$ in the quotient, a contradiction since the image of $x_{1}$ has order 3 while the image $c_{1}$ has order 2 . Similarly if $c_{1}$ belongs to $\Gamma$ then the remaining proper generators of $\Lambda$ cannot produce a nonorientable word in $\Gamma$. If $m \geq 4$ then $\mu(\Lambda) \geq \pi / 2$.

Assume thus that $s \geq 3$. If $s \geq 5$ then $\mu(\Lambda) \geq \pi / 2$. Let $s=3$. Using Theorem 2.2 once more we deduce that $\mu(\Lambda)<0$ unless $r>0$. But then $\mu(\Lambda) \geq \pi / 2$.

So it remains to consider the case $s=4$ only. By Theorem 2.2 two consecutive periods are equal to 2 . If both of the remaining periods are different to 2 then clearly $\mu(\Lambda) \geq \pi / 2$. Thus we can assume that $\Lambda=(0 ;+;[-]$, $\{(2,2,2, k)\})$. But then by Corollary $3.3 G$ is the dihedral group of order $2 k$. Thus $k=N / 2$ and consequently $\mu(\Lambda)=\pi((N-4) / 2 N)$.

Now by the formula $(2.4) N=|G|=\mu(\Gamma) / \mu(\Lambda) \leq 2 \pi(\mathbf{q}-1) / \pi((N-4) / 2 N)=$ $(4 N /(N-4))(\mathbf{q}-1)$. So $N \leq 4 \mathbf{q}$. This completes the first part of the proof.

Now given $\mathbf{q} \geq 3$ being a power of 2 let $\Lambda$ be an NEC-group with signature $(0 ;+;[-],\{(2,2,2,2 \mathbf{q})\})$ and let $G=\left\langle x, y \mid x^{2}, y^{2},(x y)^{2 \mathbf{q}}\right\rangle$ be the dihedral group $G$ of order $4 \mathbf{q}$. We define

$$
\theta\left(c_{0}\right)=x, \quad \theta\left(c_{1}\right)=1, \quad \theta\left(c_{2}\right)=(x y)^{\mathbf{q}}, \quad \theta\left(c_{3}\right)=y, \quad \theta\left(c_{4}\right)=x, \quad \theta(e)=1 .
$$

It is clear that this assignment induces a bordered surface-kernel homomorphism from $\Lambda$ onto $G$. Moreover $w=\left(c_{0} c_{3}\right)^{\mathbf{q}} c_{2}$ is a nonorientable word in $\Gamma$. Thus $\Gamma=\operatorname{Ker} \theta$ is a nonorientable bordered surface group. By (2.4) the algebraic genus of $\Gamma$ is $\mathbf{q}$. Thus $X=D / \Gamma$ is a nonorientable bordered Klein surface of algebraic genus $\mathbf{q}$ admitting the dihedral group of order $4 \mathbf{q}$ as a group of automorphisms.

A nice example of the dihedral group $D_{2 q}$ acting on a nonorientable bordered Klein surface of algebraic genus $\mathbf{q}$ can also be found in [13].

Remark. We see that $4 \mathbf{q}=8(\mathbf{q}-1)$ for $\mathbf{q}=2$ only. Thus our theorem gives a negative answer to the conjecture of May posed in [16].

Theorem 4.2. Let $G$ be a nilpotent group of automorphisms of a compact nonorientable Klein surface without boundary of algebraic genus $\mathbf{q} \geq 2$. Then $|G| \leq$ $8(\mathbf{q}-1)$. Moreover this bound may be attained only for $\mathbf{q}-1$ being a power of 2. 
Proof. Let $G$ be a finite group of automorphisms of a compact nonorientable Klein surface without boundary of algebraic genus $\mathbf{q} \geq 2$. Then $G=\Lambda / \Gamma$, where $\Lambda$ is an NEC-group with signature (2.1) and $\Gamma$ is a nonorientable surface group with $\mu(\Gamma)=2 \pi(\mathbf{q}-1)$. We will show that $\mu(\Lambda) \geq \pi / 4$.

Similarly as in the previous theorem we argue that this is so unless $g=0$, $k=1$ and $r \leq 1$, i.e. $\Lambda$ has signature

$$
\left(0 ;+;[m],\left\{\left(n_{1}, \ldots, n_{s}\right)\right\}\right)
$$

or

$$
\left(0 ;+;[-],\left\{\left(n_{1}, \ldots, n_{s}\right)\right\}\right),
$$

where all $n_{i}$ are powers of 2 .

Let us consider the first case.

If $s>2$, then $\mu(\Lambda) \geq \pi / 2$.

If $s=2$ and $n_{1}=n_{2}=2$, then $m>3$, otherwise $\mu(\Lambda)=0$, but then $\mu(\Lambda) \geq \pi / 3$. If $n_{1} \geq 4$ or $n_{2} \geq 4$ then $\mu(\Lambda) \geq \pi / 4$ and the bound is attained for $\Lambda=(0 ;+;[2],\{(2,4)\})$.

Thus let $s=1$. If $n=2$, then $\Lambda$ is generated by $x, e, c_{0}, c_{1}$ subject to the relations $x e=x^{m}=c_{0}^{2}=c_{1}^{2}=\left(c_{0} c_{1}\right)^{2}=1, e c_{0} e^{-1}=c_{1}$. In particular $c_{0}$ commutes with $c_{1}$, and generator $e$ is redundant. Since $\Gamma$ is a surface group $c_{0}, c_{1}$, and $x$ are proper generators of $\Lambda$, and since $\Gamma$ is nonorientable, a nonorientable word $w=w\left(x, c_{0}, c_{1}\right)$ belongs to $\Gamma$. Now if $w$ is nonorientable then $c_{0}$ and $c_{1}$ appear an odd number of times and using the defining relations for $\Lambda$ one can argue that $w$ is a product of a power of $x$ and one of the reflections, say $w=x^{k} c_{0}$. But then $c_{0}=x^{k}$ in the quotient $G=\Lambda / \Gamma$. Moreover $c_{1}=x^{-1} c_{0} x=c_{0}$ in $G$. So $c_{0} c_{1}=1$ in $G$, and thus $c_{0} c_{1}$ belongs to $\Gamma$. On the other hand it has order 2 , a contradiction since $\Gamma$ is a surface group. Therefore we can assume that $n \geq 4$. If in addition $m=2$ then $\mu(\Lambda)<0$. If $m=3$, then since $G$ is the direct product of its Sylow subgroups the image of $x$ and $c_{i}$ commutes for $i=1,2$. So in particular $c_{0}=c_{1}$ in $G$, and so $c_{0} c_{1} \in \Gamma$, a contradiction again. So we can assume that $m \geq 4$. But then $\mu(\Lambda) \geq \pi / 4$ and the bound is attained for $\Lambda=(0 ;+;[4],\{(4)\})$ only.

Let us consider the other case i.e. $\Lambda=\left(0 ;+;[-],\left\{\left(n_{1}, \ldots, n_{s}\right)\right\}\right)$.

If $s \geq 5$ then $\mu(\Lambda) \geq \pi / 2$.

If $s \leq 2$ then $\mu(\Lambda)<0$.

If $s=3$, and $n_{1}, n_{2}, n_{3} \geq 4$ then $\mu(\Lambda) \geq \pi / 4$ and the bound is attained for $\Lambda=(0 ;+;[-],\{(4,4,4)\})$ only, while if one of $n_{i}$, say $n_{1}=2$ then the other periods are greater than 2 , otherwise $\mu(\Lambda)<0$, but this contradicts Corollary 3.4.

Finally let $s=4$. Then $\mu(\Lambda) \geq \pi / 4$ and the bound is attained for $\Lambda=$ $(0 ;+;[-],\{(2,2,2,4)\})$ only.

We see from the proof that all groups $\Lambda$ for which the bound $\pi / 4$ is attained are generated by the elements of orders being powers of 2 . Thus since $G$ is nilpotent it must be a 2-group. 
Remark. The bound 16(q-1) (see [22]) for a nilpotent group of automorphisms of compact Riemann surface is not attained in the nonorientable case (a group of automorphisms of nonorientable Klein surface without boundary can be viewed as a group of orientation-preserving automorphisms of a Riemann surface of the same algebraic genus). It is worth noting that this is in marked contrast with the corresponding results for the maximal groups of automorphisms of compact Klein surfaces for which the bound $84(q-1)$ (in case of surfaces without boundary) and $12(\mathbf{q}-1)$ (in case with boundary) is attained both in the orientable and in the nonorientable case.

Example. Let $\Lambda$ be an NEC-group with signature $(0 ;+;[-],\{(2,2,2,4)\})$ and let $\theta: \Lambda \rightarrow G=\left\langle x, y \mid x^{2}, y^{2},(x y)^{4}\right\rangle$ be a homomorphism defined by

$$
\theta\left(c_{0}\right)=x, \quad \theta\left(c_{1}\right)=(x y)^{2}, \quad \theta\left(c_{2}\right)=y, \quad \theta\left(c_{3}\right)=x y x, \quad \theta\left(c_{4}\right)=x .
$$

Then $\theta$ is a nonorientable surface-kernel homomorphism. As a result the dihedral group of order 8 acts as a group of automorphisms on a nonorientable Riemann surface of genus 2 (i.e. the bound in the previous theorem is attained).

Remark. The above example is the only one we know and it would be interesting to know whether this case is like the nonorientable bordered one in which, as we showed in Theorem 4.1 , the bound $8(\mathbf{q}-1)$ can be improved for $\mathbf{q}>2$. Certainly if the answer to this question turns out to be affirmative another interesting problem of finding the sharp bound appears.

\section{5. $p$-GROUPS OF AUTOMORPHISMS OF KLEIN SURFACES}

Theorem 5.1. Let $G$ be a p-group $(p \neq 2)$ of automorphisms of a nonorientable bordered Klein surface of algebraic genus $\mathbf{q} \geq 2$ then $|G| \leq(p /(p-1))(\mathbf{q}-1)$. Moreover given an integer $n \geq 1$ there exists a p-group of order $p^{n}$ that acts as a group of automorphisms on a compact nonorientable bordered Klein surface of algebraic genus $\mathbf{q}=(p-1) p^{n-1}+1$.

Proof. Let $G$ be a group in question. Then $G$ can be represented as a factor group $\Lambda / \Gamma$ where $\Lambda$ is an NEC-group and $\Gamma$ is a nonorientable bordered surface group with $\mu(\Gamma)=2 \pi(\mathbf{q}-1)$. We will show that $\mu(\Lambda) \geq 2 \pi(p-1) / p$.

Since $\Gamma$ is a bordered surface group, it has a period cycle. Moreover all period cycles of $\Lambda$ are empty, otherwise since $G$ has no elements of order 2 a nonempty period cycle would induce by [1] such a period in $\Gamma$. We can assume that $\Lambda$ has a proper period, otherwise $\mu(\Lambda)$ would be a multiplicity of $2 \pi$. Moreover all periods are powers of $p$, since in the other case they would produce proper periods in $\Gamma$ [2]. If $g=0$ and $k=1$ then the reflection $c$ corresponding to this period cycle is the unique canonical generator of $\Lambda$ reversing the orientation of $D$. Moreover $c$ belongs to $\Gamma$ since $\Gamma$ has boundary. So by Theorem 2.1(ii) $\Gamma$ would be orientable. Thus either $g>0$ or $k>1$. But then $\mu(\Lambda) \geq 2 \pi(p-1) / p$, as desired. 
Now by the Riemann-Hurwitz formula (2.4) we obtain that $|G|=\mu(\Gamma) /$ $\mu(A) \leq 2 \pi(\mathbf{q}-1) / 2 \pi(p-1) / p=(p /(p-1))(\mathbf{q}-1)$. This completes the first part of the proof.

The bound $2 \pi(p-1) / p$ is attained for a group $\Lambda$ with signature $(1 ;-;[p]$, $\{(-)\})$. Let $G$ be a cyclic group of order $p^{n}$ generated by an element $A$ and let $\theta$ be the homomorphism from $\Lambda$ onto $G$ defined by $\theta(x)=A^{p^{n-1}}, \theta(c)=1$, $\theta(e)=A, \theta(d)=A^{-\left(p^{n-1}+1\right) / 2}$. Then it is clear that $\theta$ is a nonorientable, bordered, surface-kernel homomorphism. By $(2.4) \mu(\operatorname{Ker} \theta)=2 \pi(p-1) p^{n-1}$. As a result $G$ acts as a group of automorphisms on a nonorientable bordered Klein surface of algebraic genus $\mathbf{q}=(p-1) p^{n-1}+1$.

Theorem 5.2. Let $G$ be a p-group $(p \neq 2)$ of automorphisms of an orientable bordered compact Klein surface of algebraic genus $\mathbf{q} \geq 2$. Then $|G| \leq$ $(p /(p-2))(\mathbf{q}-1)$. Moreover given a positive integer $n$ there exists a p-group of order $p^{n}$ that acts as a group of automorphisms on a compact orientable surface with boundary of algebraic genus $\mathbf{q}=(p-2) p^{n-1}+1$.

Proof. Let $G$ be a group in question. Then $G$ can be represented as a factor $\Lambda / \Gamma$ where $\Lambda$ is an NEC-group and $\Gamma$ is an orientable bordered surface group with $\mu(\Lambda)=2 \pi(\mathbf{q}-1)$. Similarly as in the previous theorem we can show that $\mu(\Lambda) \geq 2 \pi(p-2) / p$.

Now by the Riemann-Hurwitz formula (2.4) we obtain $|G|=2 \pi(\mathbf{q}-1) /$ $\mu(\Lambda) \leq 2 \pi(\mathbf{q}-1) / 2 \pi(p-2) / p=(p /(p-2))(\mathbf{q}-1)$. This completes the first part of the proof.

This time the bound in question is attained for a group $\Lambda$ with signature $(0 ;+;[p, p],\{(-)\})$. From Theorem 2.0.1 in [23] we have that for any integer $n$ there exists a $p$-group $G$ of order $p^{n}$ generated by two elements $A$ and $B$ of order $p$ whose product also has order $p$. Let $\theta$ be an epimorphism from $\Lambda$ onto $G$ defined by $\theta\left(x_{1}\right)=A, \theta\left(x_{2}\right)=B, \theta(e)=(A B)^{-1}, \theta(c)=1$. Then it is clear that $\theta$ is a bordered orientable surface-kernel homomorphism. By the formula (2.4) $\mu(\operatorname{Ker} \theta)=2 \pi(p-2) p^{n-1}$ and so $G$ acts as a group of automorphisms on a bordered orientable Klein surface of algebraic genus $\mathbf{q}=(p-2) p^{n-1}+1$.

Finally in the same way as before we obtain

Theorem 5.3. A p-group of automorphisms of a nonorientable Klein surface without boundary of algebraic genus $\mathbf{q} \geq 2$, has at most $(p /(p-2))(\mathbf{q}-1)$ elements. Moreover given a positive integer $n$ there exists a p-group of order $p^{n}$ acting as a group of automorphisms on a nonorientable Klein surface without boundary of algebraic genus $\mathbf{q}=(p-2) p^{n-1}+1$.

\section{ACKNOWLEDGMENT}

The authors would like to thank the referee for comments and suggestions. 


\section{REFERENCES}

1. E. Bujalance, Proper periods of normal NEC subgroups with even index, Rev. Math. Hisp.Amer. 41 (4), 121-127.

2. _ Normal subgroups of NEC groups, Math. Z. 178 (1981), 331-341.

3. E. Bujalance and E. Martinez, A remark on NEC groups of surfaces with boundary, Bull. London Math. Soc. 21. (1989), 263-266.

4. E. Bujalance, J. J. Etayo Gordejuela, J. M. Gamboa and G Gromadzki, A combinatorial approach to automorphism groups of compact bordered Klein surfaces (in preparation).

5. J. A. Bujalance, Normal subgroups of even index in an NEC group, Arch. Math. 49 (1987), 470-478.

6. J. J. Etayo Gordejuela, Klein surfaces with maximal symmetry and their groups of automorphisms, Math. Ann. 268 (1984), 533-538.

7. J. J. Etayo Gordejuela and C. Perez-Chirinos, Bordered and unbordered Klein surfaces with maximal symmetry, J. Pure Appl. Algebra 42 (1986), 25-35.

8. N. Greenleaf and C. L. May, Bordered Klein surfaces with maximal symmetry, Trans. Amer. Math. Soc. 274 (1982), 265-283.

9. A. H. M. Hoare and D. Singerman, The orientability of subgroups of plane groups, London Math. Soc. Lecture Note Ser. 71 (1982), 221-227.

10. A. M. Macbeath, The classification of non-euclidean plane crystallographic groups, Canad. J. Math. 19 (1967), 1192-1205.

11. C. L. May, Automorphisms of compact Klein surfaces with boundary, Pacific J. Math. 59 (1975), 199-210.

12. __ Large automorphism groups of compact Klein surfaces with boundary, I, Glasgow Math. J. 111 (1977), 1-10.

13. __ A bound for the number of automorphisms of a compact Klein surface with boundary, Proc. Amer. Math. Soc. 63 (1977), 273-280.

14. __ Maximal symmetry and fully wound coverings, Proc. Amer. Math. Soc. 79 (1980), 23-31.

15. _ A family of $M^{*}$-groups, Canad. J. Math. 37 (1986), 1094-1109.

16. _ Nilpotent automorphism groups of bordered Klein surfaces, Proc. Amer. Math. Soc. 101 (2) (1987), 287-292.

17. __ Supersolvable $M^{*}$-groups, Glasgow Math. J. 30 (1988), 31-40.

18. R. Preston, Projective structures and fundamental domains on compact Klein surfaces, Ph.D. thesis, University of Texas, 1975.

19. D. Singerman, $P S L(2, q)$ as an image of the extended modular group with applications to group actions on surfaces, Proc. Edinburgh Math. Soc. 30 (1987), 143-151.

20. __ Automorphisms of compact nonorientable Riemann surfaces, Glasgow Math. J. 12 (1971), 50-59.

21. M. C. Wilkie, On non-euclidean crystallographic groups, Math. Z. 91 (1966), 87-102.

22. R. Zomorrodian, Nilpotent automorphism groups of Riemann surfaces, Trans. Amer. Math. Soc. 288 (1985), 241-255.

23. _ Classification of p-groups of automorphisms of Riemann surfaces and their lower central series, Glasgow Math. J. 29 (1987), 237-244.

Universidad a Distancia, Depto de Matematicas Fundamentales, 28040, Spain

Instytut Matematyki WSP, Chodkiewicza 30, 85-064 Bydgoszcz, Poland 